\title{
Health Technology Assessment in der Schweiz: miteinander statt gegeneinander
}

\section{Alois Gratwohl}

Prof. Dr. med. em.

Hämatologie, Medizinische

Fakultät, Universität Basel

Ein Kommentar von

Daniel Herren,

Verantwortlicher der FMH

für das Ressort Daten,

Demographie und

Qualität (DDQ) findet

sich auf Seite 1023.
Korrespondenz:

Prof. Dr. med. Alois Gratwohl Dittingerstrasse 4

CH-4053 Basel

alois.gratwohl@unibas.ch
Die Kluft zwischen unbegrenzten Wünschen und limitierten Mitteln zwingt jedes Gesundheitswesen mit Solidarprinzip zu objektivierbaren und nachvollziehbaren Entscheidungen. Health Technology Assessment (HTA) wird dafür als ein geeignetes Instrument diskutiert. Die FMH portiert das Zürcher Modell «Medical Board» unter gemeinsamer Trägerschaft mit der GDK und SAMW als HTA Konzept Schweiz. Es stellen sich Fragen zu diesem Vorgehen. Es führt inhärent zum Konflikt zwischen Bund, Kantonen und den verschiedenen Partnern im Gesundheitswesen. Das Konzept suggeriert wissenschaftliche Lösungen für politische Fragen und geht von einem HTA Modell aus, das nicht sicher Kosten spart, kaum die Qualität verbessert. Notwendig wäre eine zukunftsorientierte gesamtschweizerische Lösung.

Die Ausgaben im Gesundheitswesen steigen kontinuierlich und geben Grund zu Sorge. Moderne medizinische Verfahren können den Verlauf bisher unheilbarer Krankheiten ändern, Leiden lindern und Leben verlängern. Dies schafft Bedürfnisse und zwingt dazu, Kompromisse zwischen unbegrenzten Wünschen der Patienten und limitierten Mitteln eines solidarischen Gesundheitssystems zu finden. Die Diskussion darüber ist nicht nur in der Schweiz voll im Gange [1].

Health Technology Assessment (HTA) wurde vor etwa dreissig Jahren als neues Instrument eingeführt. Ursprüngliches Ziel war es, unwirksame oder gar schädliche Massnahmen von der Vergütung durch ein solidarisches Kostenträgermodell ausschliessen zu können [2]. Dafür wurden spezifische, unabhängige, öffentliche oder private Institutionen eingerichtet, wie das National Institute for Health and Clinical Excellence (NICE) in England oder das Institut für Qualität und Wirtschaftlichkeit im Gesundheitswesen (IQWIG) in Deutschland. Die Frage der Wirksamkeit wurde im Laufe der Zeit durch Fragen der Zweckmässigkeit und Wirtschaftlichkeit ergänzt. Der Begriff der QUALY (Quality adjusted life years), umschrieben mit der Frage «Wie viel darf ein gewonnenes Jahr Lebensqualität kosten?» fand seinen Weg ins Vokabular. Sie zu negieren, ist nicht mehr möglich [3].

Die eidgenössische Leistungskommission ELK prüft als eine ihrer Aufgaben neue Verfahren bei ihrer Einführung auf Wirksamkeit und Wirtschaftlichkeit. Im Umfeld des wachsenden Kostendrucks hat die Zürcher Gesundheitsdirektion ein «Medical Board» als kantonale HTA Institution geschaffen mit der Frage, ob und wie Kosten auch bei zugelassenen Methoden gespart werden können. Nach vier Modell-

\section{Health Technology Assessment en Suisse: unissons nos forces}

Le fossé entre volonté illimitée et moyens limités contraint tout système de santé soucieux de solidarité à prendre des décisions objectivables et claires. Pour ce faire, les HTA (Health Technology Assessments) sont considérés comme un instrument adéquat. Aux côtés de la CDS et de l'ASSM, la FMH parraine le modèle zurichois du «Medical Board» et souhaite que ce modèle serve de base à l'élaboration d'un concept HTA national. Mais la démarche suivie soulève plusieurs questions. Tout d'abord, ce concept conduit inexorablement à un conflit entre Confédération, cantons et partenaires de la santé. Par ailleurs, il propose des solutions scientifiques à des problèmes politiques, et s'inspire d'un modèle HTA qui ne garantit pas des économies de coûts et qui améliore peu la qualité. Or il serait nécessaire d'élaborer une solution nationale orientée vers l'avenir; la démarche prévue doit donc être repensée. C'est là l'occasion idéale de mettre sur pied un concept HTA moderne, comprenant une saisie des données obligatoire et prospective pour les traitements onéreux, complexes ou controversés.

Vous trouverez le commentaire de Daniel Herren, responsable du département Données, démographie et qualité (DDQ) de la FMH, sur cet article en page 1023.

untersuchungen über so unterschiedliche Themen wie Kreuzbandruptur, Avastin, Antibiotikaprophylaxe oder PET-Untersuchung soll nun das Konzept Medical Board auf die gesamte Schweiz übertragen werden [4]. Die Notwendigkeit, HTA Bestrebungen in der Schweiz zu fördern und zu koordinieren, ist gegeben. Das gewählte Vorgehen ist suboptimal. Es riskiert, Unsicherheiten und Kosten zu generieren, ohne die Qualität zu verbessern. 


\section{Organisationsstruktur}

Für die Beantwortung der einfachen Frage «Ist Verfahren X bei der Indikation Y wirksam und kosteneffizient?» benötigt es keine eigene HTA Institution in der Schweiz. Eine der anerkannten internationalen HTA Agenturen könnte die Frage schneller, kostengünstiger und neutraler beantworten. Für eine gesamtschweizerische HTA Institution umgekehrt ist die vorgesehene Trägerschaft, GDK, FMH und SAMW zu schmal. Sie schliesst wichtige Entscheidungsträger lassung durch die Privatversicherung ist wissenschaftlich nicht begründbar. Die medizinische Wissenschaft darf nicht Stellvertreterfunktion für politische Entscheide übernehmen. Die Diskussionen im Rahmen der Entscheidungsfindung für die «Hochspezialisierte Medizin Schweiz» oder um die Vergütung der alternativen Medizin zeigen nur allzu deutlich auf, wie «wissenschaftliche» Argumente oder «fehlende wissenschaftliche Grundlagen» zur Begründung politischer Entscheide nach Belieben uminterpretiert werden.

\section{Die Notwendigkeit, HTA Bestrebungen zu fördern und zu koordinieren, ist gegeben. Das gewählte Vorgehen ist suboptimal.}
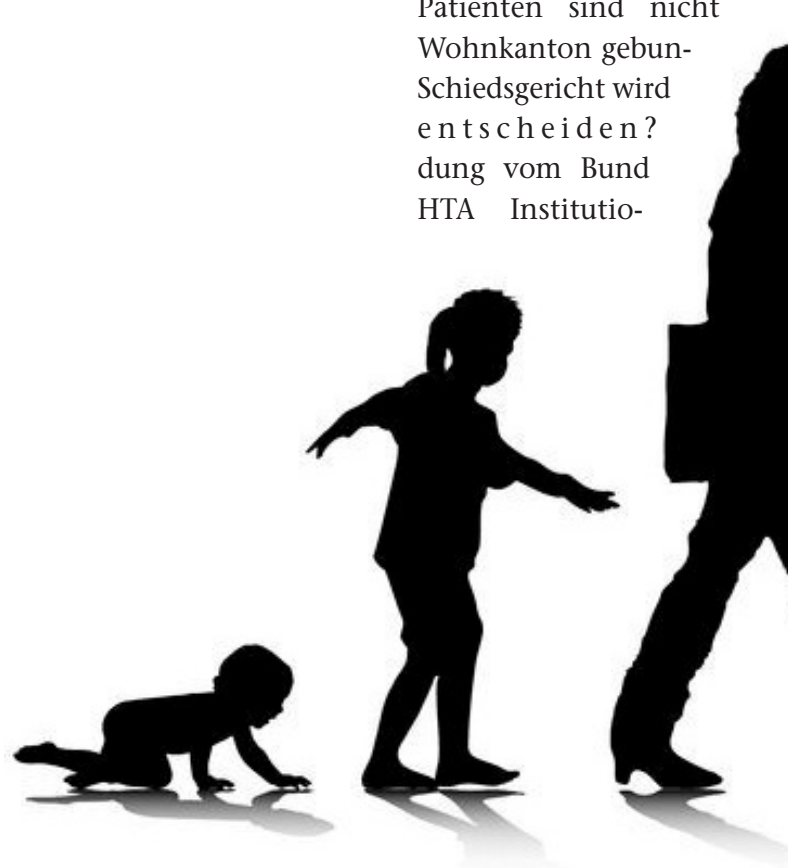

aus. Sie führt inhärent zum Konflikt zwischen Bund und den Kantonen. Komplexe medizinische Verfahren beinhalten stationäre und ambulante Kostenteile. Patienten sind nicht Wohnkanton gebunSchiedsgericht wird en $\mathrm{tscheiden?}$ dung vom Bund HTA Institutio-

?

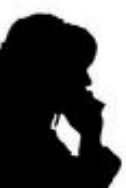
mehr an ihren den. Welches im Streitfall Ohne Einbinmit seinen nen, ohne Ein-

\section{Lehren aus der Geschichte der HTA auf internationaler Ebene}

Die anfängliche Stärke der klassischen HTA, eine Urteilsfindung aufgrund der «gesamten verfügbaren Literatur» genügt heute nicht mehr. «Evidence based medicine» hat ihre dokumentierten Stärken bei der Erstellung von Abklärungsschemata, Prophylaxe- oder Therapierichtlinien. Die Beurteilung stützt sich auf publizierte, damit immer alte Daten. Gerade bei den für eine HTA qualifizierenden kontroversen Themen riskieren laufende Studien, unberücksichtigt $\mathrm{zu}$ bleiben. Sorge bereitet die Subgruppenproblematik. Ein Ansprechen bei 30\% der Patienten mag als kosteneffizient, ein Ansprechen bei 5\% der Patienten als ineffizient beurteilt werden. Der Entscheid wird beiden Situationen nicht gerecht. Im ersten Fall wird die Mehrzahl der Patienten mit einer un-

Wie viel darf ein gewonnenes Jahr Lebensqualität kosten?

bindung der Krankenkassen, Spitäler, Pharmaindustrie und Gerätehersteller wird die Glaubwürdigkeit und Tragfähigkeit der Entscheidungen aufs Spiel gesetzt. Daten aus anderen Ländern zeigen klar auf, dass HTA Empfehlungen nur umgesetzt werden können, wenn alle national Verantwortlichen loyal zusammenstehen [5].

Jede Empfehlung für oder gegen die Kostenübernahme einer medizinischen Leistung wird zu Kontroversen führen, ihre Durchsetzung eine politische Massnahme sein. Ein Ablehnen der Kostenübernahme für Verfahren X durch die soziale Krankenversicherung unter gleichzeitiger Beibehaltung der Zu- wirksamen Therapie und damit verlorenen Kosten behandelt. Ein Verweigern einer wirksamen Therapie kann umgekehrt für die wenigen Patienten, die ansprechen würden, schwerwiegende Konsequenzen beinhalten $[3,6]$.

Aufgrund dieser erwähnten Schwächen wurde die Beurteilung nach HTA in den letzten Jahren bei ungenügender Datenlage durch ein «further studies required» ergänzt [3]. Die Umsetzung dieser Vorgabe erfolgt aber nur, wenn für die Industrie eine Chance besteht, die entstehenden Kosten zurückzugewinnen. Dies ist bei seltenen Krankheiten oder Subgruppen kaum der Fall, es sei denn, es gelingt durch die Kombination mit gezielten diagnostischen Tests als «customized therapy» einen Synergie-Gewinn zu erzielen. 
Schwieriger ist die Situation für Patienten; ihnen bleibt die Möglichkeit eines Zuganges zu der neuen Therapie vorläufig verwehrt.

\section{Prospektive obligatorische Evaluation anstelle retrospektiver Beurteilung}

Die obligatorische prospektive Erfassung neuer, vielversprechender medizinischer Verfahren bietet eine Alternative. Daten über Wirksamkeit und Wirtschaftlichkeit können in einem Evaluationsregister in der realen Alltagssituation prospektiv erfasst werden. tems. Sie wurde seit ihrer Einführung immer wieder Kosten-Nutzen-Evaluationen im Rahmen von HTAs unterworfen. Aus Kosten- und Qualitätsgründen haben sich Verantwortliche in Europa und Nordamerika auf die Erarbeitung eines Qualitätsmanagementsystems und eine standardisierte Datenerfassung geeinigt. In mehreren Ländern, auch der Schweiz, ist die Akkreditierung und obligatorische Datenerfassung Voraussetzung für die Erteilung einer Bewilligung und der Kostenübernahme. Jüngste Daten bestätigen nun, dass die Einführung des Qualitätsmangement-

\section{Für die Beantwortung der einfachen Frage «Ist Verfahren $\mathrm{X}$ bei Indika- tion Y wirksam und kosteneffizient?» ist keine eigene HTA Institution in der Schweiz nötig.}

Dies kann auch einen prospektiv randomisierten Vergleich mit der bisherigen Standardtherapie beinhalten. Voraussetzung ist, dass Kriterien für Diagnose, Entscheidungsfindung, Behandlungsart, Behandlungsdauer, Datenerfassung, Kostenabklärung und Evaluation der Studie in einem durch die zuständige Fachgesellschaft festgelegten Qualitätsmanagementsystem definiert sind.

Kosten und sogenannter «Zwangscharakter» der Massnahmen sind kein Hinderungsgrund. Daten werden schon heute gesammelt, nur unkoordiniert, an unterschiedlichen Orten. Der Aufwand ist primär organisatorischer Art. Die Kosten für die Datenkoordination werden in einem günstigen Verhältnis zu den hohen Kosten der Therapie selbst stehen. Sie sollten vom primären Kostenträger übernommen werden wie es das KVG vorsieht. Datenerfassung und Datenevaluation sind integraler Bestandteil jeder Therapie. Die Kosten werden durch Einsparungen wettgemacht. Nicht indizierte Therapien werden vermieden, Therapien ohne Ansprechen rechtzeitig abgebrochen. Der aktive Zwang, sich einem definierten Behandlungssystem anzuschliessen, ist für Patienten wie behandelnde Ärztinnen und Ärzte akzeptierbar. Er ist nur verbunden mit einer Vergütung der Kosten im Solidarsystem. Alle behalten die Freiheit, auf eine Teilnahme bei der Evaluation zu verzichten und die Kosten selbst zu übernehmen. Der aktive Zwang ist attraktiver als der passive Zwang des heute geplanten Systems, das bei unsicherer Kosten-Nutzen-Lage nur einen Ausschluss von der Kostenübernahme vorsieht.

Beispiele für das geplante Vorgehen sind vorhanden. Die Transplantation blutbildender Stammzellen ist eine anerkannte, teure Behandlungsform bei schweren Erkrankungen des blutbildenden Sys- systems in den akkreditierten Zentren mit einer signifikanten Verbesserung des Überlebens verbunden ist [7].

\section{Schlussfolgerung}

Das HTA Konzept Schweiz muss überdacht werden. Es besteht ein ideales «window of opportunity», ein modernes HTA Konzept einzuführen, mit prospektiver obligatorischer Datenerfassung für teure, komplexe oder umstrittene Therapieverfahren.

\section{Literatur}

1 Fuchs VR. Government Payment for Health Care. NEJM. 2010;363(23):2181-3.

2 Goodman CS. Introduction to Health Technology Assessment. The Lewin Group. 2004. www.nlm.nih. gov/nichsr/hta101/ta101_c1.html

3 Chalkidou K, Walley T. Using comparative effectiveness research to inform policy and practice in the UK HHS: past, present and future. Pharmacoeconomics. 2010;28(10):799-811.

4 Herren D. HTA: How to assess. Schweiz Ärztezeitung. 2011;92(14):519.

5 Mørland B, Ringard A, Røttingen JA. Supporting tough decisions in Norway: a healthcare system approach. Int J Technol Assess Health Care. 2010;Oct;26(4):398-404. Epub 2010 Oct 13

6 Elshaug AG, Garber AM. How CER could pay for itself - Insights from vertebral fracture treatments. NEJM. 2011;364(15):1390-3

7 Gratwohl A, Brand R, Niederwieser D, Baldomero H, Chabannon C, Cornelissen J, et al. Introduction of a Quality Management System and Outcome After Hematopoietic Stem-Cell Transplantation. J Clin Oncol. 2011;Apr 11. [Epub ahead of print]. 


\section{Kommentar von Daniel Herren, Mitglied des Zentralvorstandes der FMH, Verantwortlicher für das Ressort DDQ}

Keine Frage, Health Technology Assessment (HTA) steckt in der Schweiz noch in den Kinderschuhen. Und wie immer, wenn es in der Politik Neuland zu betreten gilt, versuchen sich die verschiedenen Player zu positionieren. Im konkreten Fall sind es die Initiativen des Medical Board und der SwissHTA, die das Feld derzeit besetzen.

Die einzelnen Akteure im Gesundheitswesen haben dabei unterschiedliche Interessen, wenn es um das Thema der Technologiefolgenabschätzung, wie HTA auf Deutsch auch genannt wird, geht. Die Finanzierer, Versicherungen und die Kantone möchten primär griffigere Kriterien zur Durchsetzung der WZW-Kriterien, die Ärzteschaft ist interessiert an evidenzbasierten Behandlungen, das Bundesamt für Gesundheit (BAG) will den gesetzlichen Auftrag erfüllen, und die Pharmaindustrie möchte ihre Innovationskraft behalten.

Gerade die Verfügbarkeit neuer Therapieverfahren ist für ein qualitativ hochstehendes und effizientes $\mathrm{Ge}$ sundheitswesen wichtig, allerdings stehen wir - in einem solidarisch finanzierten Umfeld - in der Verpflichtung, den Mehrwert solcher Innovationen nachweisen zu können. Hier kommen nun die Assessments zum Tragen. Mit unterschiedlichen Ansätzen versuchen die beiden erwähnten Initiativen das Thema anzugehen. Das Medical Board hat den praktischen Weg gewählt und unterzieht bestimmte medizinische Fragestellungen einer Prüfung. Man bedient sich dabei bekannter methodischer Ansätze und versucht durch die Analyse konkrete Empfehlungen zu formulieren. Interessant dabei ist, welche Implikationen diese Empfehlungen in der ärztlichen Praxis, auch ohne Sanktionierungsmassnahmen, bereits haben. Es ist allerdings nicht so, wie Kollege Gratwohl in seiner Einleitung ausführt, dass die Trägerschaft des Medical Board meint, das HTA-Konzept für die Schweiz gefunden zu haben. Die FMH engagiert sich in der Trägerschaft, um über die Fachgesellschaften den Zugang zu denjenigen Experten zu schaffen, die kompetent mitwirken können, die jeweiligen Fragestellungen inhaltlich korrekt zu begleiten.

SwissHTA, eine Initiative der Pharmaindustrie und der Versicherungen, versucht auf der Metaebene einen Konsens bezüglich ethischer Vorstellungen, methodischer Ansätze und gesetzlicher Vorgaben für die Schweiz zu formulieren. Das ist sicherlich ein wichtiger Prozess, um HTA in unserem Gesundheitssystem zu verankern. Die Initiative bietet auch die Chance, bestehende Institutionen wie zum Beispiel die Eidgenössische Leistungskommission (ELK) in ein zukünftiges HTA-Konzept zu integrieren. SwissHTA war immer als temporäre Plattform gedacht und soll sich, gemäss der Idee der Initianten, nach Erarbeitung des Konsenspapiers wieder auflösen. Grundsätzlich begrüsst die FMH eine solche Initiative, allerdings erschien uns das Vorgehen der Gründungsmitglieder von SwissHTA zu einseitig. Zwischenzeitlich hat man sich aber gegenseitig angenähert, und die FMH schaltet sich nun in die Diskussion zu diesem Konsenspapier ein.

Was uns aber vor allem fehlt, und da bin ich mit Herrn Gratwohl vollkommen einig, ist ein Leader und Koordinator all dieser Bemühungen. Wohl ist das BAG verheissungsvoll mit der Organisation eines Rundtischgespräches in die Diskussion gestartet; allerdings vermisst man seither weitergehende Koordinationsmassnahmen. Wenn man die zukunftsorientierte gesamtschweizerische Lösung erarbeiten will, wie sie Kollege Gratwohl zu Recht fordert, braucht es den Lead durch den Bund. HTA muss dabei zwingend in die übergeordnete Qualitätsstrategie des Bundes eingebaut werden, darf dabei aber kein Rationierungsinstrument werden! Ziel sollte sein, die Qualität der medizinischen Leistungserbringung zu verbessern. Im besten Fall dürfen wir eine verbesserte Ressourcen-Allokation erwarten, aber auch nur dann, wenn man sich auf die wirklich grossen Brocken der Kosten stürzt, auf die Behandlung der chronischen Erkrankungen. 